\title{
Verzeichnis der Grundrisse und Pläne
}

\author{
Aken. Stadtplan \\ Allstedt. Burg \\ Aschersleben. Stadtplan \\ Aschersleben. Stephanikirche \\ Ballenstedt. Stadtplan \\ Bernburg. Stadtplan \\ Bernburg. Schloß \\ Burgscheidungen. Schloß \\ Dessau (Mosigkau). Schloß \\ Eckartsberga. Burg \\ Eisleben. Stadtplan \\ Eisleben. Andreaskirche \\ Ermsleben (Konradsburg). Klosterkirche \\ Falkenstein. Burg \\ Frankenhausen. Stadtplan \\ Freyburg. Stadtkirche \\ Freyburg. Neuenburg \\ Frose. Stiftskirche \\ Gernrode. Stiftskirche \\ Göllingen. Klosterkirche \\ Halle. Stadtplan \\ Halle. Dom \\ Halle. Marienkirche \\ Halle. Moritzkirche \\ Halle. Moritzburg \\ Klostermansfeld. Klosterkirche \\ Kösen (Rudelsburg). Burg \\ Kösen (Schulpforta). Klosterkirche \\ Köthen. Hofkirche \\ Kyffhausen. Burg \\ Landsberg. Doppelkapclle \\ Landsberg. Doppelkapelle, Längsschnitt \\ Lauchstädt. Situationsplan \\ Mansfeld. Schloß \\ Memleben. Plan des Klostergeländes \\ Merseburg. Dom \\ Naumburg. Stadtplan \\ Naumburg. Dom \\ Naumburg. Wenzelskirche \\ Naumburg. Marientor \\ Nienburg. Klosterkirche \\ Oranienbaum. Lageplan von Stadt und Schloß \\ Oranienbaum. Stadtkirche \\ Petersberg. Klosterkirche
}


Quedlinburg. Stadtplan

Quedlinburg. Stiftskirche St. Scrvatius

Quedlinburg. Stiftskirche St. Wiperti

Quedlinburg. Benediktikirche

Quedlinburg. Blasiikirche

Querfurt. Burg

Sangerhausen. Klosterkirche

Schwenda. Dorfkirche

Seeburg. Burg

Stecklenberg. Lauenburg

Weißenfels. Schloß

Wendelstein. Burg

Westerhausen. Dorfkirche

Wettin. Burg

Wittenberg. Stadtplan

Wörlitz. Park

Zeitz. Lageplan von Dom und Schloß 